\title{
Photon gluon fusion cross sections at HERA energy
}

\author{
J.J. Engelen, S.J. de Jong, M. Poletiek, J.A.M. Vermaseren \\ NIKHEF-H, 1009DB Amsterdam, The Netherlands
}

Received February 1988; in revised form 29 March 1988

\begin{abstract}
We present cross sections for heavy flavour production through photon gluon fusion in electron proton collisions at HERA energy. The electron photon vertex is taken into account explicitly, and the $Q^{2}$ of the exchanged photon ranges from nearly zero (almost real photon) to the kinematically allowed maximum. In our approach the scale is set by the mass of the produced quarks. Our formalism is also applicable to the production of light quarks as long as the invariant mass of the pair is sufficiently high, so we also give cross sections for $u \bar{u}, d \bar{d}$ and $s \bar{s}$ production.
\end{abstract}

\section{Introduction}

In the framework of perturbative QCD, photon gluon fusion (Fig. 1a) is the only mechanism contributing at the Born term level to the (virtual) photoproduction of heavy flavours, i.e. charm and beyond. In fact, the masses of the quarks involved suggest considering heavy flavour production as a short distance phenomenon and thus a description in terms of perturbative QCD. This approach is proposed and followed in [1]. Photon gluon fusion involving virtual photons has been described in [2] and [3]. It has been successfully used to describe multi-muon muoproduction [4]. Photon gluon fusion, extended with additional assumptions, can also be relevant to the description of $J / \psi$ photoproduction. The latest experimental results, compared to one particular model, are reported in [5], where further references to other experimental results and theoretical models, all related to photon gluon fusion, can be found.

In this paper we carry out calculations for the kinematical configuration of HERA, the electron proton collider presently under construction at the DESY laboratory in Hamburg, where $30 \mathrm{GeV}$ electrons and $820 \mathrm{GeV}$ protons will collide head on. The purpose of these calculations is twofold. First of all we want to determine the cross section for $t \bar{t}$ production, as a function of the top mass, in order to establish up to which mass photon gluon fusion leads to observable top production. Furthermore the bottom and charm production rates are also of interest as both bottom and charm physics are interesting areas of research.

Secondly, photon gluon fusion could constitute a background to deep inelastic $e p$ scattering at HERA. Indeed, more generally speaking, photoproduction of jets (photon gluon fusion, QCD Compton scattering, processes involving the anomalous photon structure function and other higher order processes) will have a considerable cross section at HERA. (A first rather qualitative discussion of QCD Compton scattering as a background at HERA can be found in [6]). It will be important to develop a reliable, quantitative picture of these photon induced processes as has been done for fixed target photoproduction $[7,8]$. Such work should, of course, not only be inspired by background considerations but also by the quantitative tests of perturbative QCD offered by these processes.

\section{The model}

The diagram that we consider in this paper is given in Fig. 1b. We take the photon propagator into account exactly, i.e. we do not use the WeizsaeckerWilliams approximation for the equivalent photon beam. so we can give the cross section as a function of the virtual photon invariant mass squared, $-Q^{2}$.

The gluon is extracted from the target proton according to a fractional momentum $(x)$ distribution given by the gluon structure function. For this structure function we either take the properly normalised form prescribed by dimensional counting, $G(x)=$ $3(1-x)^{5} / x$, or a scale dependent expression $G\left(x, S^{2}\right)$ for which several parametrisations are proposed in the literature [9]. The scale $S^{2}$ we define as $S^{2}=M^{2} / 2$, where $M^{2}$ is the invariant mass squared of the $q \bar{q}$ pair that is produced. This scale $S^{2}$ is also used for the running strong coupling constant $\alpha_{s}=\alpha_{s}\left(S^{2}\right)$. As mentioned before our results refer to $30 \mathrm{GeV}$ electron on $820 \mathrm{GeV}$ proton collisions.

Our choice of scale provides a natural cut off for 
$a$

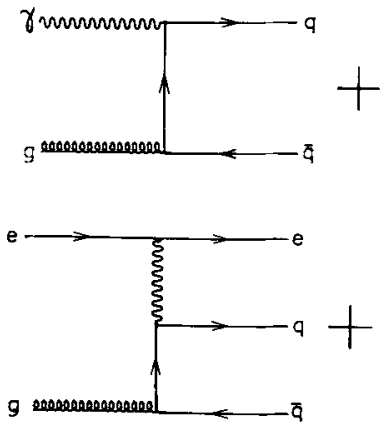

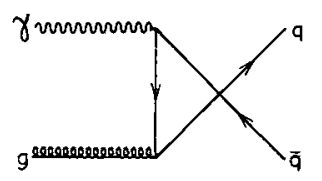

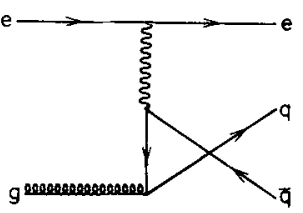

Fig. 1.a Feynman diagram for photon gluon fusion in lowest order perturbation theory. b Feynman diagram for the photon gluon fusion process in electroproduction

heavy quarks. For light quark production we will integrate from a minimal value of $S$ in order to justify the application of perturbative QCD.

\section{Top production}

In Table 1 we give the cross sections we find for $t \bar{t}$ production, for several values of the top mass. Results are given both for the counting rule gluon structure function and for the scale dependent gluon structure function as parametrised by Eichten et al., version 2 [9e]. Other parametrisations give results very similar to the EHQL parametrisation, with the exception of [9a] which is known to have too steep an $x$ dependence. The results obtained with the scaling violating structure function are the more realistic ones. We include the cross section values corresponding to the counting rule structure function to demonstrate the sensitivity of the result to the 'hardness' of the gluon distribution, an effect that is more pronounced for a larger top mass. This result can be easily understood because there is a kinematical threshold on $x: x$ $>M_{t i}^{2} / s$, with $s$ the centre of mass energy of the collision.

The top production cross section as a function of the top mass is displayed in Fig. 2. In the same figure we show the top production cross section from $W$ gluon fusion as presented in [14].

In Fig. 3 the cross section is given as a function of

Table 1. Total cross section for $t \bar{t}$ production through photon gluon fusion at HERA energy $(\sqrt{s}=314 \mathrm{GeV})$ for the counting rule and Eichten et al. set 2 gluon distribution functions respectively. Results are given for various top masses

\begin{tabular}{lll}
\hline & & $\sigma(\mathrm{pb})$ \\
$m_{t}(\mathrm{GeV})$ & Counting rule & E.H.L.Q. set 2 \\
\hline 40 & 4.77 & 2.15 \\
45 & 2.47 & 0.97 \\
50 & 1.30 & 0.45 \\
55 & 0.70 & 0.21 \\
60 & 0.37 & 0.10 \\
65 & 0.20 & 0.048 \\
70 & 0.11 & 0.022 \\
\hline
\end{tabular}

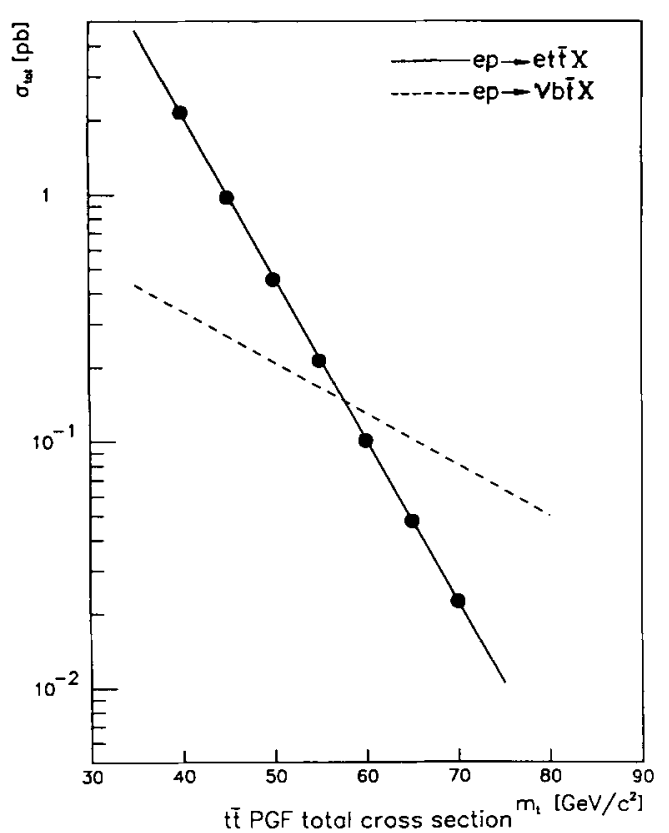

Fig. 2. Total $t \bar{t}$ production cross section for photon gluon fusion at HERA energy $(\sqrt{s}=314 \mathrm{GeV})$ as a function of the top mass The gluon distribution parametrisation is as given by Eichten et al. (full dots). The full line is drawn to guide the eye. The dashed line is the total cross section for top production through $W$ gluon fusion at HERA energy as calculated by Baur and Van der Bij [14]

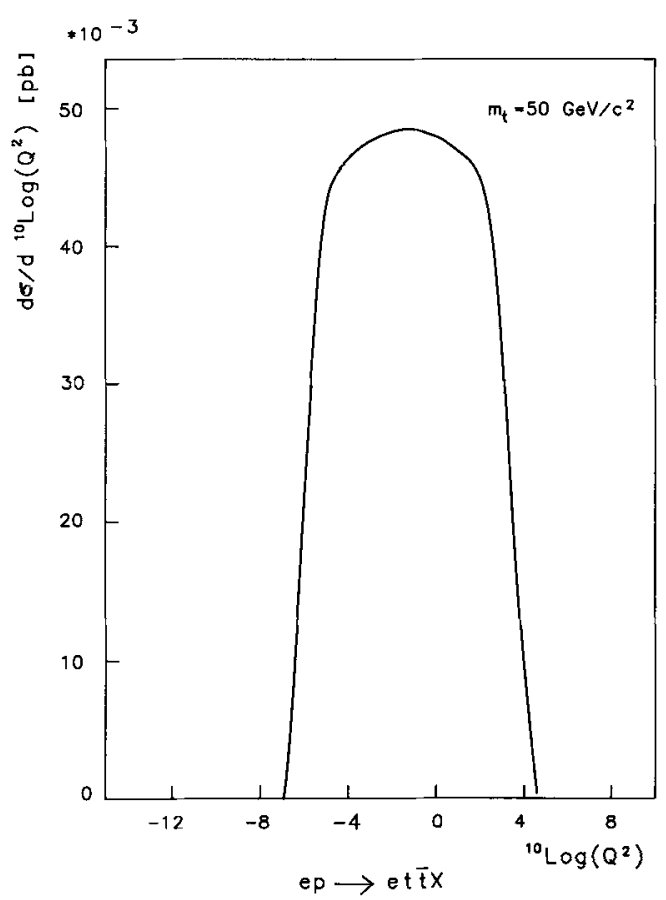

Fig. 3. Differential cross section for $t \bar{t}$ production through photon gluon fusion as a function of ${ }^{10} \log \left(Q^{2}\right)$. The gluon distribution parametrisation is as given by Eichten et al. The top mass is taken to be $50 \mathrm{GeV} / \mathrm{c}^{2}$ 
$Q^{2}$. Note the logarithmic scale of the $Q^{2}$ axis. A large fraction of the cross section corresponds to $Q^{2}<$ $1 \mathrm{GeV}^{2}$ (photoproduction), but a sizable fraction corresponds to $Q^{2}>1 \mathrm{GeV}^{2}$, up to the kinematic limit (deep inelastic leptoproduction). It is clear that adequate detection efficiency over this large kinematical range will impose very high requirements on the performance of HERA detectors.

From Table 1 as such it is not possible to derive a discovery limit for the top mass. This would require a detailed simulation study applied to a specific detector, which is not the aim of this paper. In the past a discovery limit of $0.1 \mathrm{pb}$ has been postulated for exotic processes at HERA [13], i.e. for processes with a rather unique signature. If we apply this criterion to our results (table 1) we arrive at a discovery limit of $\sim 65 \mathrm{GeV}$. (We multiply the numbers in Table 1 by a factor of 2, because 2 top particles are produced per event). This is not the ultimate limit that can be reached at HERA. It has been shown [14] that $W$ gluon fusion pushes this limit up to about $85 \mathrm{GeV}$. For top masses up to $55 \mathrm{GeV}$, however, photon gluon fusion will dominate and give rise to reasonable numbers of events for an integrated luminosity of $200 \mathrm{pb}^{-1}$.

\section{Charm and beauty production}

In table 2 we present the cross sections for $c \bar{c}$ and $b \bar{b}$ production. For the $c$ quark we give results both for a quark mass of $1.5 \mathrm{GeV} / \mathrm{c}^{2}$ and of $1.6 \mathrm{GeV} / \mathrm{c}^{2}$. The results are rather sensitive to the value of this parameter. For the $b$ quark a mass of $5 \mathrm{GeV} / \mathrm{c}^{2}$ is assumed. Again we include the results obtained with the counting rule gluon structure function for comparison and to indicate that, in principle, heavy flavour production gives a handle on the gluon structure function. Contrary to top production, charm and beauty production are enhanced for a 'softer' gluon distribution, because the threshold effect mentioned above plays practically no role for the relatively much lighter $c$ and $b$ quarks. In Figs. 4 and 5 we show the $Q^{2}$ spectrum for $b \vec{b}$ and $c \bar{c}$ production, respectively. The cross sections we find are large. The integrated luminosity of $200 \mathrm{pb}^{-1}$, quoted above, would correspond to 80 million $c \bar{c}$ and to more than 800 thousand $b \bar{b}$ events. To what extent any particular experiment is capable of detecting these processes will have to be investigated separately. Apart from the physics

Table 2. Total cross section for $b \bar{b}$ and $c \bar{c}$ production through photon gluon fusion at $\sqrt{s}=314 \mathrm{GeV}$ for the counting rule and the Eichten et al. set 2 gluon distribution function respectively

\begin{tabular}{lcc}
\hline & & \multicolumn{2}{c}{$\sigma($ nb) } \\
& Counting rule & E.H.L.Q. set 2 \\
\hline$c \bar{c}\left(m_{c}=1.5 \mathrm{GeV} / \mathrm{c}^{2}\right)$ & 354 & 429 \\
$c \bar{c}\left(m_{c}=1.6 \mathrm{GeV} / \mathrm{c}^{2}\right)$ & 296 & 375 \\
$b \bar{b}\left(m_{b}=5.0 \mathrm{GeV} / \mathrm{c}^{2}\right)$ & 2.8 & 4.3 \\
\hline
\end{tabular}

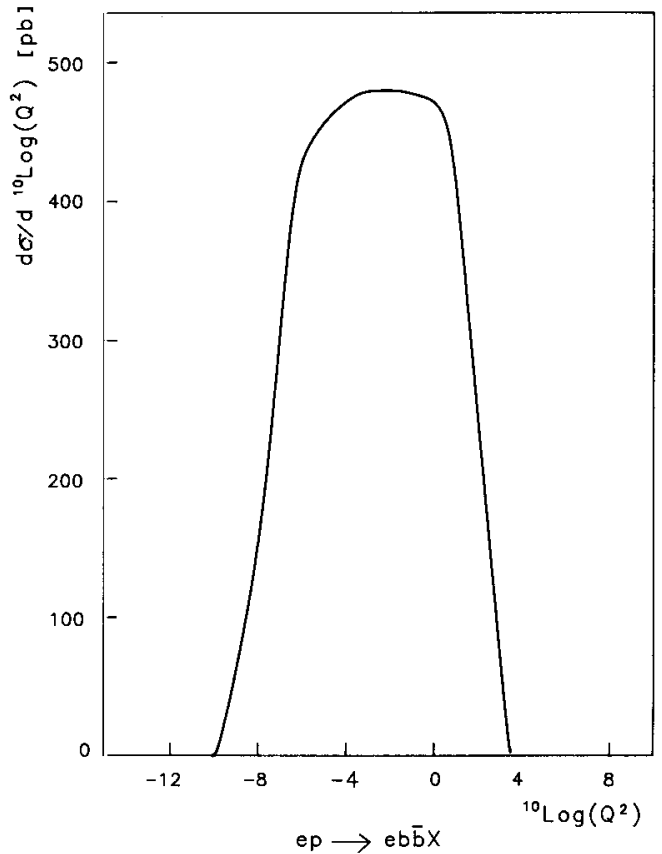

Fig. 4. Same as Fig. 3 for $b \bar{b}$ production

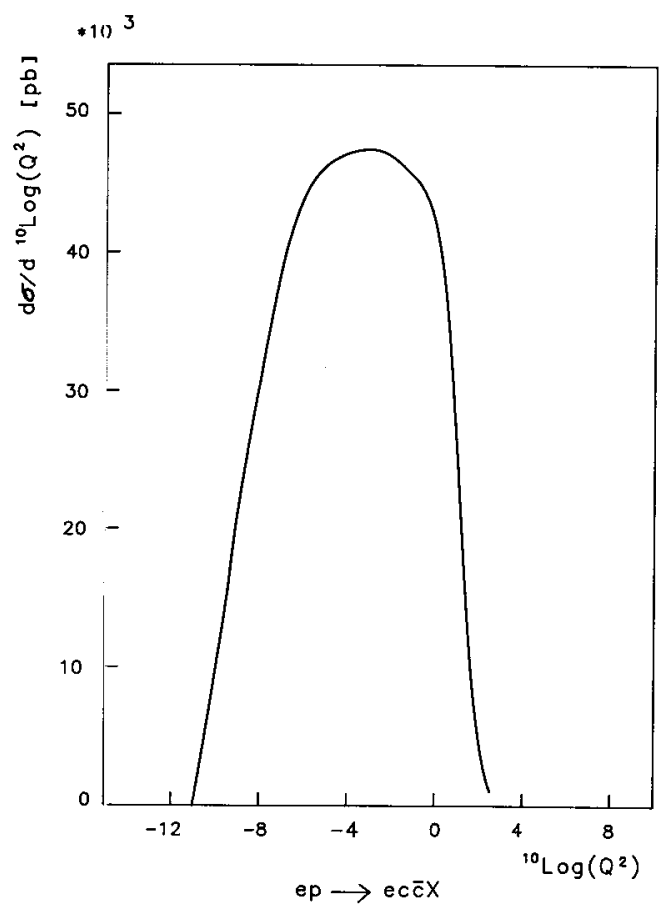

Fig. 5. Same as Fig. 3 for $c \bar{c}$ production

potential (gluon structure function, spectroscopy, quark mixing), also the possible background of these processes to standard deep inelastic charged current and neutral current scattering and in particular to 'exotic phenomena' has to be a point of concern. 


\section{Light quark production}

In Table 3 we give the results on $u \bar{u}, d \bar{d}$ and $s \bar{s}$ production through photon gluon fusion. The cross sections are given for three different values of the cut off on the $q \bar{q}$ invariant mass, as indicated in the table. The main reason for presenting these cross sections is their large value. So far we have not investigated the possibility of experimentally exploiting these processes for physics analysis. In any case these reactions are an important source of particle production at HERA and therefore possibly a source of background.

\section{Potential background from photon gluon fusion}

The following example illustrates the mechanism by which a background to deep inelastic scattering can develop. For a $q \bar{q}$ pair produced in photon gluon fusion one or more of the hadronisation products are unobserved because they remain inside or close to the beam pipe. Such particles are emitted at small angles with respect to the collision axis but can be quite energetic and have substantial transverse momentum. In the case of heavy flavour production the unobserved high transverse momentum particle might be a neutrino from semi-leptonic decay. At the same time the final state electron will, with high probability remain in the beam pipe and escape observation (Fig. 6).

Such an event will look like a deep inelastic charged current event.

In order to obtain a quantitative estimate of this type of contamination the complete final state has to be studied. To generate final states we first split up the final state quarks originating from the proton into a spectator quark-diquark system. The fraction of the incident proton momentum assigned to the quark follows the distribution

$$
x_{q}=\left(1-x_{g}\right) \sqrt{(1-x)^{3} / x}
$$

according to [10]. $x_{g}$ is the proton momentum fraction carried by the initial state gluon. The momentum fraction of the diquark is then $x_{Q}=1-x_{q}$.

We subsequently employ the LUND fragmentation scheme [11] for hadronising the two colour singlet systems resulting from a combination of the produced

Table 3. Total cross section for $s \bar{s}\left(m_{s}=0.5 \mathrm{GeV} / \mathrm{c}^{2}\right) d \bar{d}\left(m_{d}=\right.$ $\left.0.325 \mathrm{GeV} / \mathrm{c}^{2}\right)$ and $u \bar{u}\left(m_{u}=0.325 \mathrm{GeV} / \mathrm{c}^{2}\right)$ production through photon gluon fusion at $\sqrt{\mathrm{s}}=314 \mathrm{GeV}$ for the Eichten et al. set 2 gluon distribution function at various values for the quark-antiquark invariant mass squared cut-off

\begin{tabular}{lccl}
\hline & & $\sigma(n b)$ & \\
& $M_{q \bar{q}}>2 \mathrm{GeV} / \mathrm{c}^{2}$ & $M_{q \bar{q}}>3 \mathrm{GeV} / \mathrm{c}^{2}$ & $M_{q \vec{q}}>4 \mathrm{GeV} / \mathrm{c}^{2}$ \\
\hline$u \bar{u}$ & 2660 & 1300 & 828 \\
$d \bar{d}$ & 664 & 325 & 207 \\
$s \bar{s}$ & 524 & 277 & 176 \\
\hline
\end{tabular}

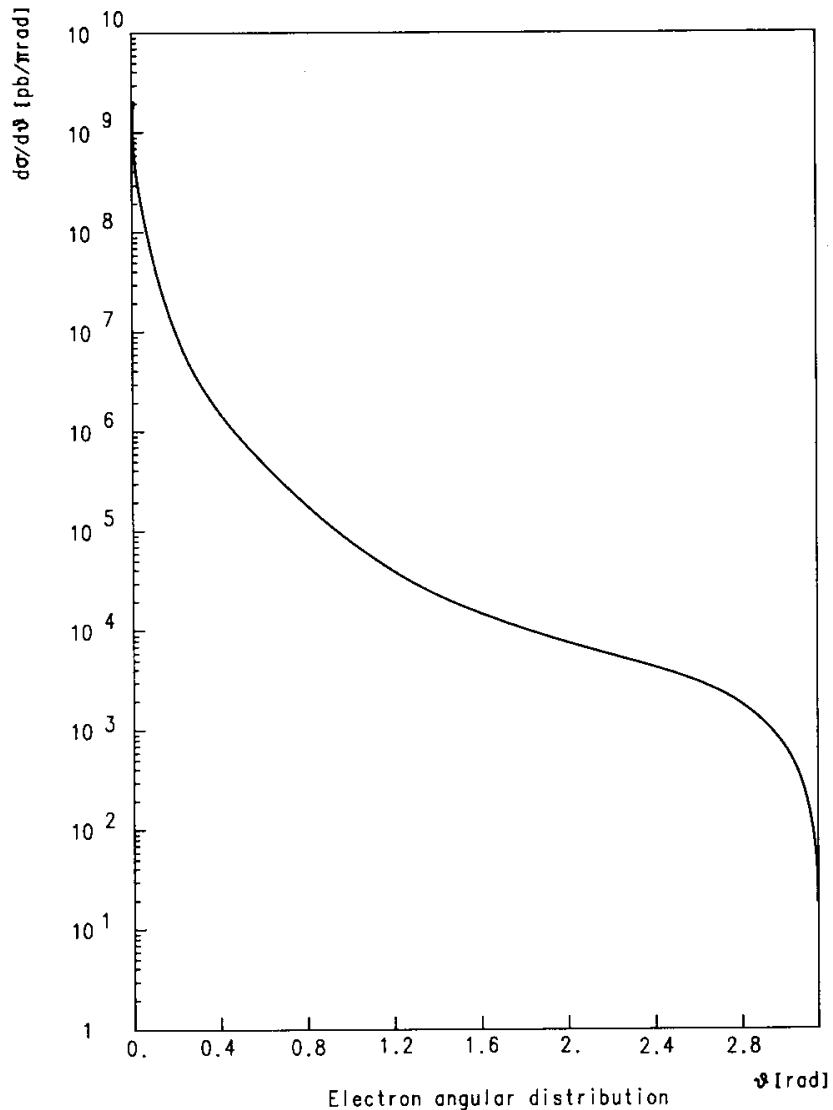

Fig. 6. Differential cross section for $u \bar{u}$ production through photon gluon fusion as a function of the polar angle of the electron in the final state with respect to the incoming electron

quark (Fig. 1) and the spectator diquark, and the produced anti-quark (Fig. 1) and the spectator quark, respectively.

A priori a photon gluon fusion event and a charged current event will look quite different. In the former case the $q$ and $\bar{q}$ jets will be back to back in the plane perpendicular to the collision axis for the majority of the events, i.e. the low $Q^{2}$ events for which the final state electron is not deflected appreciably with respect to the collision axis. We assume that this electron is not observed. (The high $Q^{2}$ events with an observed electron will obviously not contaminate the charged current sample).

Jet fragments emitted at a small angle with respect to the beam axis will escape detection. These particles can be energetic and can carry non negligible transverse momentum in spite of the small production angle. Applying the so called Jacquet Blondel method [12] to such events, discarding the non-observed particles, will then lead to a $Q^{2}$ measurement which yields too high a value. The structure of these events (jet at small angle with energetic leading particle) will render the recognition and detection of both jets difficult or impossible. 
Similar arguments apply to certain events with missing energetic neutrinos from e.g. charm decay.

It turns out that a convolution of these rather rare hadronisation and/or decay configurations with the high production cross sections leads to an a priori non negligible contamination of the charged current sample. A quantitative analysis requires the simulation of the acceptance of a specific detector. Preliminary results of such a quantitative analysis show that a contamination can be expected up to $Q^{2}$ of about $500 \mathrm{GeV}^{2}[15]$.

A completely different kind of contamination can be caused by heavy flavour production. Heavy flavour production can lead to multi-lepton final states. Neutrinos will lead to the observation of missing energy. Such events might mimic final states originating from "exotic processes", of which examples can be found in [13]. We want to emphasize that experimental observation of certain low cross section exotic processes leading to final states as sketched above will require a quantitative analysis including photon gluon fusion over the entire $Q^{2}$ range.

\section{Conclusions}

Cross sections for heavy and light quark production in electron proton collisions have been presented. The calculations are based on the photon gluon fusion diagram and are performed in the leading log approximation of perturbative QCD. Moreover the electron-photon vertex is taken into account explicitly.

Top quark production through this process should be observable up to a top quark mass of $65 \mathrm{GeV}$. It is dominated by photon gluon fusion for top quark masses below $55 \mathrm{GeV}$, above this value $W$ gluon fusion will dominate. (This latter process will in fact push the discovery limit of top to a value of about $85 \mathrm{GeV}[14])$.

Very large cross sections are found for $c \bar{c}$ and $u \bar{u}$, $d \bar{d}$ and $s \bar{s}$ production. The $b \bar{b}$ cross section turns out to be large as well. These large cross sections, especially for heavy flavour production, imply a large physics potential. They also mean that photon gluon fusion, if not taken into account carefully, can be a source of background for other, lower cross section processes to be studied at HERA.

Acknowledgement. We thank J. van der $\mathrm{Bij}$ for useful discussions.

\section{References}

1. L. Jones, H. Wyld: Phys. Rev. D17 (1978) 759; H. Fritzsch, K.-H. Streng: Phys. Lett. 72B (1978) 385

2. J. Babcock, D. Sivers, S. Wolfram: Phys. Rev. D18 (1978) 162

3. M. Glück, E. Reya: Phys. Lett. 83B (1979) 98; J. Leveille, T. Weiler: Nucl. Phys. B147 (1979) 147

4. EMC Collab. J.J. Aubert et al.: Phys. Lett. B1 10(1982) 73; EMC Collab. J.J. Aubert et al.: Nucl. Phys. B213 (1983) 1; EMC Collab. J.J. Aubert et al.: Nucl. Phys. B213 (1983) 31

5. NA14 Collab. R. Barate et al.: Z. Phys. C-Particles and Fields 33 (1987) 505

6. J.J. Engelen: Proc. of the Workshop 'Experimentation at HER A', Amsterdam (1983) 241

7. P. Aurenche et al.: Phys. Lett. 135B(1984) 164; P. Aurenche et al. Z. Phys. C24 (1984) 309; D.W. Duke, J.F. Owens: Phys. Rev. D26 (198:) 1600

8. NA14 Collab. P. Astbury et al.: Phys. Lett. 152B (1985) 419; NA14 Collab. E. Auge et al.: Phys. Lett. 168B (1986) 163

9. A.J. Buras, K.J.F. Gaemers: Nucl. Phys. B132 (1978) 249; 9 b M. Glück, E. Hoffmann, E. Reya: Z. Phys. C-Particles and Fields 13 (1982) 119; 9c D.W. Duke, J.F. Owens; Phys. Rev. D30 (1984) 49; 9d R. Field, R.P. Feynman: Phys. Rev. D15 (1977) 2590; 9e E. Eich en, I. Hinchliffe, K. Lane, C. Quigg: Rev. Mod. Phys. 54 (1984) 579; E. Eichten, I. Hinchliffe, K. Lane, C. Quigg: Rev. Mod. Phys. 58 (1986) 1065

10. A. Capella, J. Tran Thanh Van: Z. Phys. C-Particles and Fields 10 (1 781) 249; A. Capella and J. Tran Thanh Van: Z. Phys. CPart cles and Fields 18 (1983) 85

11. T. Sjostrand: Comp. Phys. Commun. 27 (1982) 243 and references therein

12. R. Turlay: DESY $79 / 48$ (1979) 393

13. R.J. Cashmore: Proc. of the Workshop 'Experimentation at HERA', 381. Amsterdam 1983

14. U. Biur, J.J. van der Bij: CERN-TH $4875 / 87$ (1987)

15. S.J. de Jong, J.J. Engelen: to appear in the Proc. of the Workshop 'Physics at HERA', Hamburg (1987) 